\title{
ADVANCES IN STUDIES ON NATURAL PRESERVATIVES FOR FRUITS AND VEGETABLES
}

\author{
Haisheng Gao *, Pengbao Shi, Yuhua Zhao \\ Department of Food Engineering, Hebei Normal University of Science \& Technology, \\ Changli, Hebei Province, P.R. China 066600 \\ * Corresponding author, Address: Department of Food Engineering, Hebei Normal University \\ of Science \& Technology, Changli, 066600, Hebei Province, P.R. China, Tel: +86-0335- \\ 2039374,Fax:+86-0335-2039374,Email:spxghs@163.com
}

Abstract: The author introduced general research and application situations of natural preservatives for fruits and vegetables all over the world these years, and summarized application of vegetation of Murraya in Rutaceae, Cinnamomum in Lauraceae, Artemisia in Compositae and other families and genera on fruits and vegetables preservation and fresh-keeping. Decoction or extraction of Chinese traditional medicine, such as Alpinia Officinarum, Amarphalus Konjac K., stemona etc, could be used in fresh-keeping for orange, apple, strawberry, edible fungi and so on. Garlic could be used in fresh-keeping for orange. Phytic acid and fresh-keeping agents compounded with Phytic acid could extend storage periods of easily rotting fruits and vegetables, such as strawberry, banana, cantaloup, edible fungi and so on, and better keep original fresh condition. Extraction of Snow Fresh, Semper Fresh, Arthropod shell extraction, and halite also had better effect on preservation and fresh-keeping for fruits and vegetables. Main problems exsited in the application of natural preservatives for fruits and vegetables were showed in this article and the applying prospect were discussed too.

Keywords: fruits, vegetables, fresh-keeping agent, fresh-keeping effect, applying prospect

Fruits and vegetables fresh-keeping agents could be divided into two categories, i.e. compound and natural fresh-keeping agents. For a long time, people mainly used compound substance as fresh-keeping agent to keep stored fruits and vegetables fresh. Although this method had better effect on

Please use the following format when citing this chapter:

Gao, H., Shi, P. and Zhao, Y., 2009, in IFIP International Federation for Information Processing, Volume 295, Computer and Computing Technologies in Agriculture II, Volume 3, eds. D. Li, Z. Chunjiang, (Boston: Springer), pp. 1655-1669. 
fresh-keeping of stored fruits and vegetables and preventing them from rotting, many compound substance had bad effects to man's health, and might lead to cancer, distortion and virulence mutation(Gao Haisheng,2002; Yi Ming,2006). So people began to pay attention to developing and researching natural fresh-keeping agents, and had made a great progress.

\section{ANTISEPSIS AND FRESH-KEEPING OF EDIBLE FLAVOR VEGETATION}

The ancients had applied antisepsis and fresh-keeping function of edible flavor vegetation for a long time. Hoffman and Evans found that mustard seeds, clove and cassia all had the effects of preventing fruits and vegetables from rotting. Adding $0.5 \mathrm{~g}$ mustard seeds in $100 \mathrm{~g}$ apple juice, this could keep its quality for 4 months. The reason why edible flavor vegetation had antisepsis function was that there was active matter-essential oil in it(Lin Jinneng,1991;GaoHaisheng,1993). Ripptter and Wise approved that essential oil in mustard seed, clove, cassia bark, elettaria cardamomum, caraway seed, allspice and thyme all had antisepsis function to a certain degree. Essential oil existed in vegetation root, stem, leaf, flower and fruit. For instance, clove bud contained essential oil by $15 \% \sim 20 \%$, and $85 \% \sim 92 \%$ of it was eugenol. It was eugenol that had main antisepsis function. Similarly, Chinese cassia contained essential oil by $1.0 \% \sim 3.5 \%$, and $80 \% \sim 95 \%$ of it was cinnamal; Sri Lanka cassia contained essential oil by $0.5 \% \sim 3.5 \%$, and $42 \% \sim 75 \%$ of it was cinnamal. Cinnamal was the active ingredient.

\subsection{Vegetation of murraya in rutaceae}

Essential oil in branch and leaf of Murraya almost could control and kill familiar mould. They were ideal raw materials of natural fresh-keeping agents for fruits. Vegetation of Zanthoxylum were widely used as food anticorrosive, had strong controlling and killing function to fruit malignant disease-producing germ. Essential oil(especially not mature essential oil) in Citrus vegetation peel had very strong controlling and killing function to Penicillium digitiatum and Penicillium italicum causing orange blue and green mould. This discovery led to success in researching natural freshkeeping agent for orange, and a national patent was obtained.

\subsection{Vegetation of cinnamomum in lauraceae}

Most of vegetation of Cinnamomum were arbores, its root, stem, branch and leaf contained rich essential oil. In essential oil contained a great deal of 
cinnamal, borneo camphor, 1,8 $\sim$ cineole, eugenyl methyl ether, linalool and so on, and these could control and kill microbe. Eugenyl methyl ether, cinnamal, and linalool could be used in fruit fresh-keeping experiments.

\subsection{Other vegetation}

Besides the vegetation mentioned above, many other flavor vegetation had active matter of controlling and killing function to fruit malignant diseaseproducing germs. For instance, ascitriodora, eucalyptus, blue gum of Eucalyptus in Myrtaceae; tsaoko, curcuma of Amomum and Curcuma in Zingiberaceae were widely used as food anticorrosive and fresh-keeping agents. In addition, there were citronella, lemongrass, rue grass in Poaceae; acorus gramineus soland, anise calamus in Araceae; perilla, sweet basil in Labiatae.

\section{EFFECTS OF EXTRACTION FROM CHINESE HERBAL MEDICINE VEGETATION ON FRUITS AND VEGETABLES FRESH-KEEPING}

Many vegetation could be used as medicine. The useful ingredients extracted by decocting and immersing were used to treat fruits and vegetables combining with other drugs, and got better effect on fresh-keeping.

\subsection{Amarphalus Konjac K. Extraction}

Tuber of Amarphalus Konjac K. contained rich konjaku flour, the main ingredients in it were konjaku mannoside, konjaku mannoside content was $50 \%$, and konjaku mannan could be obtained after extracted using organic solvent. konjaku mannan had no color, innocuity and no peculiar smell. It had certain function to fruit fresh-keeping and antisepsis of fish and meet. One treatment was as follows: fresh strawberries were putted in $0.05 \%$ konjaku mannan solution and doused for 10 minutes, taken them out, redundant sugar fluid was dripped, and dried in the air. The strawberries used this treatment would lose a little gloss after stored 1 week under room temperature, but they would not mildew and rot, and even not mildew after stored for 3 weeks. On the contrary, untreated strawberries would lose their gloss after stored 2 days under room temperature, and start to mildew and rot after 3 days(Zhu Liangfeng et al.,1991).

\subsection{Decoction of Alpinia Officinarum}

The main antisepsis component in Alpinia Officinarum was naphtha, 
occupying $0.5 \% \sim 1.5 \%$ and including $1,8 \sim$ cineole, methyl cinnamate, clove oil, phenol pinene, cubeben etc. Its main piquancy component was galangol, and main yellow components were galangin, galangin, and kaempferide.

Preparation and application of Alpinia Officinarum decoction were as follows: $1 \mathrm{~kg}$ Alpinia Officinarum and $10 \mathrm{~kg}$ water were put together and decocted for 45 minutes. Water was being supplemented at any time to supplement evaporated water during boiling. Be sure that the mixture was approximately being kept $10 \mathrm{~kg}$. The obtained mixture was filtered when it was hot, then cooled for using. The obtained decoction and bleaching (decoction : bleaching $=1: 1.5$ ) were put together and adjusted to coating. Oranges were painted on their surfaces and encased in wicker baskets, and stored in rooms under normal temperature. Or the painted oranges were dried in the air, wrapped in $0.01 \mathrm{~mm}$ thickness plastic films with little holes $\left(0.25 \mathrm{~mm}^{2}\right)$, and stored under normal temperature. After stored for 95 days, the treated oranges rotting rate was only $7.8 \%$, and untreated oranges rotting rate was 37\%(Yi Ming,2006;Zhu Liangfang et al.,1991).

\subsection{Allium Sativum extraction}

Allium Sativum contained garlic naphtha, the main component in garlic naphtha was garlicin. It came into being through garlic amino acid in fresh garlic being decompounded by garlic enzyme. Allicin had controlling and killing function to fungus, such as anthracnose fungi, damping-off fungi and rhizopus nigricans. Preparation of garlic extraction was as follows: Fresh garlic pieces were soaked in cold water for 12 hours, then decocted to boiling, finally $10 \%$ garlic extraction was made for using. Or 1 portion of triturated garlic and 10 portions of $80 \sim 90^{\circ} \mathrm{C}$ water were put together and cooled to normal temperature. One using method was that picked oranges were soaked in garlic extraction, taken out after $10 \sim 15$ minutes, dried in the air, put in fir boxes with empty cases and hardboards, and stored in ventilated storehouses or common rooms. Good fruits rate after stored for 70 days was over 92.4\%(Gao Haisheng,1990a).

\subsection{Phytic Acid fresh-keeping agent}

Phytic Acid was a sort of organic acid widely existing in vegetation seeds. Fruits and vegetables anticorrosive made using Phytic Acid could be used in antisepsis and fresh-keeping of easily rotting fruits and vegetables and edible fungi. Some melons and fruits, such as grape, strawberry, Hami melon, banana, ananas and litchi could be painted with the agents. This could not only maintain their faint physiological activity, ideal air permeability and water permeability, but also raise melon and fruit shine, enhance resisting ability of outside germs invading, and observably inhibit enzyme activity. Edible fungi could be treated with Phytic Acid, this could prevent edible fungi from changing color, solve the problem of $\mathrm{SO}_{2}$ remaining, extend edible fungi fresh period from $2 \sim 3$ days to $5 \sim 7$ days. This method also worked in fresh cherry, which was difficult in 
storing(Wu Fan,2003).

According to L zuyou's experiments in strawberry fresh-keeping(Luo Zuyou et al.,2002), the obtained parameter constitution through orthogonal selection was $0.1 \% \sim 0.15 \%$ Phytic Acid $+0.05 \%$ sorbic acid $+0.1 \%$ acetic hyctro peroxide. Strawberries treated with the mixture fluid could be kept fresh for 1 week under normal temperature, and 15 days under low temperature, good fruit rate was $90 \% \sim 95 \%$. In addition, Phytic Acid could keep fruits quality. Dissolubility solids, organic acid and Vc content in Strawberries would be raised after treated with $0.1 \%$ or $0.15 \%$ Phytic Acid. The reason was that Phytic Acid had anti-oxidation effect. Another report was that Phytic Acid could efficiently prevent fruits and vegetables from brown changing and $\mathrm{Vc}$ losing in processing.

\subsection{Chinese traditional medicine compound semipermeable film fresh-keeping agent}

Stemona, giant knotweed rhizome, galangal rhizome, berberine and so on could be extracted under exceed critical condition, the extraction was mixed with starch, konjaku and lecithin, finally Chinese traditional medicine compound semipermeable film fresh-keeping agent was obtained. Some formulas were as follows:

In formula I (Zhu Xuan,2001;Kong Qiulian et al.,2002), included 0.5 $2.0 \mathrm{~g} / \mathrm{L}$ starch, $0.5 \sim 1.0 \mathrm{~g} / \mathrm{L}$ galangal rhizome, $0.5 \sim 1.0 \mathrm{~g} / \mathrm{L}$ stemona, $0.5 \sim$ $1.0 \mathrm{~g} / \mathrm{L}$ giant knotweed rhizome, $0.5 \sim 1.5 \mathrm{~g} / \mathrm{L}$ konjaku, $0.5 \sim 1.0 \mathrm{~g} / \mathrm{L}$ berberine and $0.1 \sim 2.0 \mathrm{~g} / \mathrm{L}$ lecithin. The apples treated with the mixture could be preserved for 6 months; tomatoes, mad apples and cucumbers treated with it could be stored for 2 months under $10 \sim 15^{\circ} \mathrm{C}$.

In formula II (Song Xiaogang et al.,1996), included 50g Chinese prickly ash, $50 \mathrm{~g}$ cassia bark, $50 \mathrm{~g}$ clove and $300 \mathrm{ml}$ starch oxide. $20 \mathrm{~g}$ starch, $0.12 \mathrm{~g}$ $\mathrm{KMnO}_{4}, 0.15 \mathrm{~g}$ borax, $1 \mathrm{~g} \mathrm{NaOH}$ and $100 \mathrm{ml}$ water were put together, heated to $90 \sim 95^{\circ} \mathrm{C}$, continuously stired for $20 \sim 30$ minutes, cooled it, and then starch oxide was obtained. At first, the mixture was even painted on common packing paper to form a continuous and even Chinese herbal medicine painting layer. The layer was baked under $60 \sim 80{ }^{\circ} \mathrm{C}$ to form compound fresh-keeping paper. The Ya pears stored in it under room temperature for 35days would be kept better fresh condition and nourishment.

In formula III(Song Xiaogang,1997), included 33g stemona, 33g giant knotweed rhizome, $54 \mathrm{~g}$ galangal rhizome, $14 \mathrm{~g}$ licorice and $270 \mathrm{ml}$ starch oxide. Compound fresh-keeping paper was made according to the method mentioned above. Tomatoes, sweet peppers and green cucumber were wrapped in pieces of this paper, then stored under $26 \sim 34^{\circ} \mathrm{C}$, relatively 
humidity $75 \% \sim 98 \%$, and deposited in no direct sun light places. This could diminish nutrient losing. The tomatoes wrapped in this films would be basically kept fresh after stored for 18 24 days, except their color became a little dark, and untreated tomatoes were completely rotted. Sweet peppers stored in this films for $12 \sim 18$ days didn't rot, except their color had a changing period of green, yellow and red. Green cucumber wrapped in this films would be kept its fresh for about $6 \sim 12$ days.

Formula IV (Wei Qin,1998), i.e. NFP fresh-keeping agent, included modified $0.3 \%$ konjaku glucan, $0.2 \%$ CMC-Na and $0.1 \%$ garlic extraction. Scatheless oranges were put in NFP fresh-keeping extraction and soaked for $1 \sim 2$ minutes, then took them out and stored under room temperature. The orange rotting rate was $25 \%$ after stored for 60 days, weight losing rate was $3.0 \%$, and they kept yellow and shining surface. Untreated oranges rotting rate was $50 \%$, weight losing rate was $3.7 \%$, their surface was lackluster.

\subsection{Application of Nicandra Physaloides(L.) Gaertn seed extraction}

Nicandra Physaloides(L.) Gaertn seed extraction had good film forming character. The film had no color and no smell, dissolved in water rapidly and washed easily. So we can use it in fruit and vegetable fresh-keeping.

Jinguan apple, early ripening variety, painted with $0.5 \% \sim 1 \%$ the extraction would not change its surface color and basic quality after stored for 40 days under $25 \sim 30{ }^{\circ} \mathrm{C}$ room temperature in open places. But the untreated apples would became yellow in 2 weeks, and when stored to 40 days, they would be wizened seriously and lose perfume and merchandise value. Using the same extraction to treat middle or late ripening varieties would had better effects. Ya pears painted with $0.25 \% \sim 0.5 \%$ the extraction and stored for 40 days in open places under room temperature would not be changed in quality, and the untreated pears would become wizened, and quality and merchandise value decreased. Similarly, using $0.5 \% \sim 1 \%$ the same extraction to paint fruits and vegetables, such as sweet peppers, cucumber and tomatoes could efficiently prolong their shelf life for over 10 days. Vc content of the sweet peppers painted with $1 \%$ the extraction and stored for 15 days was $1.364 \mathrm{~g} / \mathrm{kg}$, still at high level, and $\mathrm{Vc}$ content in untreated ones was only $1.047 \mathrm{~g} / \mathrm{kg}$ 。

Fresh-keeping mechanisms of Nicandra Physaloides(L.) Gaertn seed extraction were as follows: after painted with the extraction, there would form a even and colorless film on fruit surface. This provided a small air conditioning environment. It efficiently controlled fruit respiration metabolizing, postponed cell becoming decrepit and pigment degrading, 
and extended fruit fresh-keeping time. Different varieties of fruits had different tolerance to $\mathrm{CO}_{2}$, so different varieties of fruits had different ideal treating concentration. The experiments results showed that apple ideal treating concentration was $0.5 \% \sim 1 \%$, Ya pear and Xuehua pear ideal treating concentrations were $0.25 \% \sim 0.5 \%$. Sweet pepper, other fruit and vegetable ideal treating concentrations were $0.5 \% \sim 1.0 \%$ (Zhu Xiangqiu et al.,1997).

After painted with the extraction, the fruit surface gloss was enhanced. It had glazing and waxing function, so the extraction as a fresh-keeping agent was very suitable in fruits and vegetables fresh-keeping in shelf period.

\section{PHOSPHOPROTEIN HIGH MOLECULAR PROTEIN FRESH-KEEPING MEMBRANE}

Phosphoprotein high molecular protein widely existed in animals and vegetation. In protein molecular contained a lot of hydrophilic group, such as $-\mathrm{NH}-,-\mathrm{O}-,-\mathrm{COOH},-\mathrm{OH}$ and $-\mathrm{NH}$. After forming film, phosphoprotein high molecular protein fresh-keeping membrane had suitable air permeability, water permeability and gas selectivity. After soaked in phosphoprotein high molecular protein, in fruit surface would form even film, and its thickness could be easily adjusted among tens micron according to fruit physiological development. The film could observably control respiration intensity of fruits and vegetables(Guan Wenqiang et al.,2006). Hygienic toxicity experiments result showed that little albino rat oral LD of phosphoprotein high molecular protein was over $10000 \mathrm{mg} / \mathrm{kg}$, it belonged to no poison medicines.

Its using concentration should be $3 \% \sim 7 \%$, pH value was $2.7 \sim 8.5$. On fruits and vegetables surface would form films in 3 hours after they were soaked. General dosage was $0.15 \sim 0.5 \mathrm{~kg} / \mathrm{t}$. Storing experiments result showed that Good fruit rate of Jingguan apples stored for 5 months was 95\%, and Good fruit rate of Guoguang apples stored for 6 months was $98 \%$. It had better effects in orange and banana. The experiment result in strawberry showed that strawberry treated using concentration of $1 \% \sim 2 \%$ would be prolonged its store time for 2 days under normal temperature, and $15 \sim 20$ days under $4 \sim 8^{\circ} \mathrm{C}$ (Guan Wenqiang et al.,2006). 


\section{NATURAL FRUIT AND VEGETABLE FRESH- KEEPING AGENT STUDIED ABROAD AND STARTED}

\subsection{Snow fresh}

Snow Fresh was a new-style high effect multiple function fruit and vegetable fresh-keeping agent made in MONSANTO chemical company in America. It could defer oxidization and brown changing of fruits and vegetables. It had better effect on fresh-keeping of fruit and vegetable, semimanufactured goods, which had no peel and pit. According to experiments, Snow Fresh could at least keep semi-manufactured goods color and organization shape for 5 days, and the effect was better than that of sulfite which commonly used at present. Snow Fresh contained four safe and avirulent components, i.e. sodium pyrophosphate, citric acid, ascorbic acid and CaCL. Snow Fresh was different from sulfite, which had blanching function. Fruits and vegetables treated with sulfite and stored for some time not only had obvious peculiar smell, but also had rudimental sulfur in them. Fruits and vegetables treated with Snow Fresh had no peculiar smell.

Snow Fresh using methods were simple and convenient. Under room temperature, the fixed quantity of Snow Fresh (white powder) was added in water, mixed for $1 \mathrm{~min}$, and $1 \% \sim 3 \%$ solution was compounded. Fruit and vegetable soaking time was $0.5 \sim 3$ min. Snow Fresh solution should be made and used in one day, and supplemented Snow Fresh powder in it to keep its concentration to get optimal effects(Gao Haishengb,1990).

\subsection{Semper fresh}

Semper Fresh was a sort of fruit and vegetable fresh-keeping agent made in SEMPER Genetic Engineering Company in England. It had such characteristics as no color, no smell, no virulence, no pollution and no side effect. The edible Semper Fresh was widely used in fruit and vegetable fresh-keeping, and succeeded in flower fresh-keeping. Semper Fresh was a chemical compound, containing vegetable oil and sugar, its active component was sugar ester, other components were cellulose, edible oil etc. Its fresh-keeping mechanism was that it could control fruits and vegetables respiration, prevent water evaporating in order to make them rest, slow them aging speed. Its using concentration was $0.8 \% \sim 1.0 \%$ for smooth peel melon and fruit, proper higher concentration for rough peel fruit, and proper lower concentration for strawberries and leaf vegetables. The concrete using concentrations were $1.0 \%$ (variation between $0.8 \% \sim 1.0 \%), 0.8 \%, 1.0 \% \sim$ 
$1.5 \%$ and $1.2 \%$ for apples, pear (no stipe), orange and banana respectively. In addition, the fresh-keeping experiments in strawberry, cherry and apricot using Semper Fresh were done in Shanxi agricultural science academy, and the effects were ideal. Grape fresh-keeping was usually carried out in grape trees (when the grapes were $70 \sim 80 \%$ ripeness) with $0.8 \% \sim 1.0 \%$ concentration Semper Fresh, then picked after $3 \sim 4$ days. The using concentration was $0.6 \% \sim 1.0 \%$ for melon, about $0.8 \%$ for tomato, and $0.8 \% \sim 1.0 \%$ for fresh kidney beens and pepper. Semper Fresh could be stored in cool and dry places for a long time. Blended solution could be stored for 5 days. According to different proportions of agent and water, Semper Fresh was dissolved in water and deposited over one night for using. This could assure the powder pervading completely. Selected fruits and vegetables were soaked in Semper Fresh solution for 30 seconds, then taken them out and dried in the air. In a general way, $1 \mathrm{~kg}$ Semper Fresh powder could treat 28 35t apples(Gao Haisheng,1990; Wu Fan,2003).

\subsection{Arthropod shell extraction}

Arthropod shell extraction was generally called chitosan. It was reported(Gao Haisheng,1990c;Zhu Dongxing,2003) that its main component was derivative of deacetylated chitin, a sort of cation high molecular polysaccharide. The extraction was safe and innoxious. It could be washed away by water, degaded by creature. It had no rudimental toxicity problem. The semipermeable film forming on fruit surface could adjust fruits physiological metabolizing(Ghaouth,1992a), and control many microorganisms(Ghaouth,1992b). Using the compound membrane to control fruits and vegetables air condition and slow them physiological metabolizing had been already reported(Asahi,1981,1982,1983). The compound film segregating gas factors could be expressed as $a \mathrm{a} / \mathrm{b}=\mathrm{Pa} / \mathrm{Pb}$. Among them "p" stands for pervious ability coefficient, "a" and "b" stand for different gases. For the film containing hydroxyl molecular, a $\mathrm{CO}_{2} / \mathrm{O}_{2}<1$, it had better effect on fruits and vegetables fresh-keeping. For general compound film, a $\mathrm{CO}_{2} / \mathrm{O}_{2}>1$, it had no fresh-keeping function to fruits and vegetables. Chitosan cames into being from chitindeacetylation, so its molecular contained amino-group as well as hydroxyl group. Its forming compound film segregating gas factors could be expressed as, a $\mathrm{CO}_{2} / \mathrm{O}_{2}<1$. If metal ions were added in chitosan, $a$ a/b value would be much smaller(Bai,1988), so it was more suitable to fruits and vegetables freshkeeping. Du chuanlai et al.(2004)reported that fixed quantity of chitosan $(50 \% \mathrm{NaOH}$ deacetylation) was dissolved in $2 \%$ acetic acid solution, added in $0.05 \%$ metal chloride salt solution, stired for 30 minutes, finally chitosan 
painting agent containing metal ion was prepared. It had better effect on grape fresh-keeping under normal temperature. Among the treatments, the extraction containing $\mathrm{Ca}^{2+}$ had the best effect on fresh-keeping, grape rotting rate and fresh weight losing rate were both the lowest.

EL-Ghaouth et al.(1983)found that using $0.1 \%$ deacetylated chitin to paint strawberries could greatly inhibit rotting of them stored under $13{ }^{\circ} \mathrm{C}$. After 21 days, rotting rate was about $1 / 5$ that of untreated strawberries, the effect was better than germicide. It had no harm to strawberries, and better kept them hardness. Yang yuan'er(2002)uesd 2\% chitosan to treat cucumbers, the fresh-keeping period lasted 20 days long, and the color was not changed. Sweet peppers treated with chitosan could be stored for 25 days or much longer. The experiments in amaranth, Chinese chive, greens also displayed fresh-keeping effects at different degrees. Although it worked to bananas and apples, but not to wild peaches, even accelerated them deteroprating. Neutral solution of N, O-carboxymethylated-chitosan extracted from shrimp and crab was used to treat Huaguang 2 Kivifruit, single fruit was painted to form membrane on it, wrapped in polyethylene bag, in the bag $\mathrm{KMnO}_{4}$ ethylene absorbefacient was added. Yangtao used this kind of treatment could be stored for 3 months under normal temperature, good fruit rate was $100 \%$, and weight losing rate was only $3 \%$.

chitosan dissolving capability was better in thin acid, and $\mathrm{H}^{+}$ concentration was $1 \sim 100 \mathrm{nmol} / \mathrm{L}$. In order to easily control acidity, some organic acids, sush as Ctiric Acid and Acetic Acid, were used as menstruum. Chitosan doesn't dissolve in water, so its using is not convenient. Chitosan water-solubility ramification A was developed in Hebei Agricultural University, it had more powerful inhibiting bacterium function. The lowest inhibiting concentrations to sporeine, penicilla, rhizopus nigricans, main various food harmful microorganisms were all $1 \%$. The product had stable inhibiting function under high temperature and high pressure(Qi Guohui et al.,2004).

\subsection{Compound Ve ramification fresh-keeping agent}

American scientists found that $\mathrm{Vc}$ ramification and its chemical compound could keep opened apples not to become brown for 48 hours. Components of this kind of compound $\mathrm{Vc}$ ramification included $\mathrm{Vc}$ ramification (for instance, Ascorbic acid -2- Phosphate or Ascorbic acid-6aliphatic acid), cassia acid, $\beta$-cyclodextrin, sodium phosphate and so on. It could be used as fresh-keeping agent after fruits were flayed and before processing, replacing sulfite in can, preserved fruit, confection, and fruit juice drink producing. It was widely used as a agent of no social effects of pollution and not influencing products flavor(Gao Haisheng,1990d). 


\subsection{Halite extraction}

Halite extraction was a sort of substance extracted from mineral in rock layer. It was white powder, mainly containing metal-salt of $\mathrm{Ca}, \mathrm{P}, \mathrm{Mg}, \mathrm{Na}, \mathrm{Mn}$ and so on, and used as fresh-keeping agent for fruits and vegetables. When using, put $10 \mathrm{~g}$ halite extraction in $75 \mathrm{~L}$ water and wait until it dissolved. Experiment results indicated that the agent had significant effects in easily rotting fruits and vegetables fresh-keeping, such as strawberry, arbutus, mushroom and so on. Its another advantage was that halite extraction could decompose chemical fertilizer and agricultural chemicals on the surface of fruits and vegetables, and kill parasite eggs(Wu Fan,2003).

\section{PROBLEMS AND THE VIEW OF APPLICATION}

\subsection{Problems}

\subsubsection{The lack of systematic theoretical guide and fundamental studies}

There are several steps in the study of natural preservatives for fruits and vegetables: the distillation and separation of substance that can effectively restrain germ growth, the characteristic of the substance in medium, the application test in fruits and vegetables preserving of the substance, the toxic evaluation, etc. So the development of new natural preservatives requires multidisciplinary knowledge as its basis, such as food science, microbiology, biochemistry, medicine, Chinese traditional medicine, etc. However, researchers in these fields seldom collaborate. On the other hand, there is a general lack of enthusiasm for developing new preservatives among enterprisers because of their unwillingness to invest in fundamental studies. Thus, the development of new natural preservatives for fruits and vegetables is limited in some degree.

It's known that the extractives of many plants possess the property of antisepsis, and many of them have already been marketed as natural preservatives. However, most of these preservatives are crude products, and concentrations of the effective species vary with season and geographical environments. For some natural preservatives, the identification of chemical structures for its compositions has not been carried out thoroughly. Consequently, the active species are still unknown and its separation and toxicology evaluation can not be performed. As for the action mechanisms, 
antimicrobial spectrum, application domains, and the relationship between molecular structure and its activity, it is still unclear for most new natural preservatives.

\subsubsection{Problems in film coating for fresh-keeping}

Because of its own structural limitations, polysaccharides have defects in several aspects of film coating such as the moisture retention and antibacterial action. Thus, it is necessary to add functional additives and active substances or to alter the characteristics of polysaccharides, so as to make them capable of antisepsis.

It is essential to take care on each step during application of the film coating reagents, for factors such as film concentration and thickness, marinating time and apparatus are all influencing for the final results. It is difficult to determine the thickness of the film and the concentrations of the reagents, because the surface roughness vary with breeds and batches.

On the other hand, film coating is not suitable for all produces. Some produces are capable of self-protection because there is a layer of fuzz on their surfaces. It is likely that the fuzz be destroyed by film coating and the storage time shortened.

\subsubsection{Problems in natural products applications}

The essential oils have been industrialized as pesticide, but they are still not industrialized as antibacterial agent and they are barely used in preservatives for fruits and vegetables. The studies of mechanism and applied technology are not thorough yet. And so much studies of these fields are in the test period and the mature products are absence.

The reports about natural products for fresh-keeping of the bacteriostasis test are all carried through in medium. The results showed that the bacteriostasis concentration should be increased in the production application. While the essential oils are all hydrophobic compound, they can influence the flavor of fruits and vegetables. Besides the studies mostly processed in the conditions that environment invariety and single microorganisms exist. Lack in the studies that the combination and application of natural extractives on antisepsis characteristic.

There are still some problems though the major studies are about bacteriostasis test of plant crude extract. One of the problems is that the extraction and seperation do not proficiency. The second problem is that the extraction methods are variable and the structure of active ingredient can not be ascertained easily. The last one is that the bacteriostasis mechanism still dimness and studies on applied technology are not enough yet. 


\subsection{View of applying prospect}

Rencently years, peoples are focuse on the studies and developments about organism polysaccharides and then green fresh-keeping methods became the hot point in the domain. The development of polysaccharides film will centralize in the following aspects in future. One of it is to confirm the coat structure ,moisture content, polysaccharides concentration and marinating time by mathematical model.The second one is presentation fresh-keeping mechanism of the polysaccharides film-coating. The third one is combining the research results with production to enhance the industrialization degree.

Internationally, the trend in development of preservatives for fruits and vegetables is natural and functionality. What is more, studies and application about it will more and more. But it is still in start-up periods in china. Resently years, many natural preservatives have been empoldered under the active endeavor of scientific research institutions and manufacturers. Some of the products are spread the application positively and achieved good results.

With the development of food industry and advance in living standard and awareness raising, the security of some synthetic preservatives have been questioned. For these reasons, hunting safe and efficient natural preservatives became the focus point in food industry domain. Developing botanical and natural antiseptic for fruits and vegetables turns into the first choice according to the security, resource and economics because there are extensive territory and abundance on plant resource in china. Most of the materials are good taste, abundant nutrition and health function that can be used as food and medicinal materials or condiment in the same time normaly. They can endow special aroma to fruits and vegetable, moreover, they are low mammalian toxicity. Such antiseptics easier to be accepted and they appease the demand for green food. So it is not only the mainstream development in abroad but also the major aspect in china. Though botanical and natural antiseptic impossible replace the chemosynthesis one entirely because the restrain of price and bacteriostasis effect, the chemosynthesis one must be substitute in future for the technology advancement, living standard improving and safety consciousness gather head more.

\section{REFERENCES}

Asahi Dow Ltd.,J. P. Patent,1981,78,968.

Asahi Dow Ltd.,J. P. Patent,1982,63,25.

Asahi Dow Ltd.,J. P. Patent,1983,415,925.

Bai R. K. Polymer Bulletin 1988,20,83. 
Du Chuanlai, Wang Jiahong, Yu Zhifang. Research and application of natural preservrtive of fruits and vegetables. Science and Technology of Food Industry,2004,25(5):135-138(in Chinese)

EL-Kazzaz, M K et al . Effect of different atmospheres on postharvesy decay and quality of fresh strawberries. Photopathology,1983,73:282-285

Gao Haisheng. Edible spice plant and application of it[J]. World Agriculture,1993,(5):2326(in Chinese)

Gao Haisheng. Natural preservatives for fruits and vegetables-snow fresh. Shan Xi Fruit Trees, 1990b,(3):57(in Chinese)

Gao Haisheng. New method to prevent fruits broening. Chinese Agricultural Science Bulletin,1990c,(2):9(in Chinese)

Gao Haisheng. New methods for fruits fresh-keeping.[J] Herbei Agricultural Science and Technology,1990d,(1):31(in Chinese)

Gao Haisheng. Production and application of natural fresh-keeping agents for fruits and vegetables[J]. The Farmers Consultant,2002,(8):19(in Chinese)

Gao Haisheng.Introductions about several natural fresh-keeping agents for fruits and vegetables[J]. World Agriculture,1990a,(4):44-46(in Chinese)

Ghaouth A E,Hortscience,1992a,27(9):1016.

Ghaouth A. E. Phytopathnology,1992b,82(4):398.

Guan Wenqiang, Li Shufen. Research advances in application of natural plant extracts to post-harvest preservation of fruits and vegetables[J]. Transactions of the Chinese Society of Agricultural Engineering,2006,22(7):200-204(in Chinese)

Kong Qiulian, Zhang Mingrong, Zhang Lili. Inhibition on aspergillus niger and penicillium expansun of Chinese medicinal herb[J]. Storage and Process,2002,(3):17-18(in Chinese)

Lin Jinneng.Edible spice plant and application of it[M]. Beijing: Light Industry Press,1991,28-30. (in Chinese)

Luo Zuyou, Luo Shunhua. Preliminary probe into the applying organic acids compound freshkeeping agents to strawberry storage[J]. Hubei Agricultural Sciences,2002,(3):67-69(in Chinese)

Qi Guohui,Nan Yan, Li Baoguo,etc.Effect of free-polluntant fresh-keeping agents on Taihang chestnut (Castanea mollissima Bl.)[J]. Journal of Agricultural University of Hebei,2004,27(1):53-56(in Chinese)

Song Xiaogang, Chen Min, Wu Yahong. Studies on the fresh-keeping effect of several Chinese medicinal herb and compound preservative paper for juicy pear [J]. Food Science, 1996,17(2):67-69(in Chinese)

Song Xiaogang, Chen Min, Wu Yahong. Studies on the fresh-keeping effect of small packing of Chinese medicinal herb compound preservative paper[J].Food Science,1997,18(2):5457(in Chinese)

Wei Qin.Manufacture of natural preservative for orange preservation and its effect[J]. Science and Technology of Food Industry, 1998,(2): 32-33(in Chinese)

Wu Fan. Application of natural fresh-keeping agents for fruits and vegetables in abroad[J].Shan Dong Food Science and Technology,2003,(4):28-30(in Chinese)

Yang Yuan'er, Luo Faxing.A new crude food preservative-chitosan[J]. Journal of Wuhan Polylechnic University, 2002,21(3):22-25(in Chinese)

Yi Ming. Studies on natural fresh-keeping agents for fruits and vegetables[OL]. China Net on Applying of Food Additives,2006.1.10(in Chinese)

Zhu Dongxing, Li Shengyin. Summarization of applying studies on preservatives for fruits and vegetables[J].Shan Xi Jurnal of Agricultural Science,2003,(1):30-34(in Chinese)

Zhu Liangfeng, Lu Biyao. Studies on natural fresh-keeping agents for fruits[J].Letters in China Food Additives, 1991,4:32-35(in Chinese) 
Zhu Xiangqiu, Wei Jianmei, Mao Zhenchuan.The coloid characteristc of seeds of saloides (L). Gaertn and its application on food industry.Colloquium collection of essays about international food process and packing technology[C].Beijing:Engineering Industry Publishing House, 1997(in Chinese)

Zhu Xuan. Advance in using natural Chinese herb as fruits and vegetables preservatives[J]. Xinjiang Agricultural Sciences,2001,41(Special Issue):110-111(in Chinese) 